\title{
Biobanking metastases and biopsy specimens for personalized medicine
}

This article was published in the following Dove Press journal:

Journal of Biorepository Science for Applied Medicine

7 October 2015

Number of times this article has been viewed

\author{
Angen Liu \\ Charles C Collins \\ Stephanie M Diemer \\ Sidney Kimmel Comprehensive \\ Cancer Center, Johns Hopkins \\ University School of Medicine, \\ Baltimore, MD, USA
}

Correspondence: Angen Liu Biospecimen Repository, Sidney Kimmel Comprehensive Cancer Center, Johns Hopkins University School of Medicine, 4 I 7 North Carolina Street, Room 302, Baltimore, MD 2I 287, USA

$\mathrm{Tel}+\mathrm{l} 4105023195$

Email angenliu8@gmail.com
Abstract: Metastasis often develops in cancer patients despite improvements in surgery, chemotherapy, and radiation therapy. Biopsy specimens of metastatic lesions are extremely valuable materials for monitoring disease progression, assessing therapeutic outcome, and delivering personalized medicine. Biobanking high-quality human metastases and biopsy tissue specimens, derivatives, liquid biopsy specimens, and associated patient clinical information provides a fundamental scientific infrastructure for personalized medicine. Biomarkers specifically associated with an individual tumor have a very significant impact on personalized therapy. Therefore, the availability of a large collection of metastases and biopsy specimens from cancer patients with well-annotated patient clinical and pathological data is a critical requirement for personalized medicine. While current efforts have been made to develop cancer biorepositories around the world for personalized medicine, the majority of biospecimens in these biorepositories are primary tumor samples. The procurement, processing, and storage of human metastases and biopsy specimens remain challenging, since metastatic tumors are almost incurable and most are not the object of surgical resection. Thus, biobanking metastases and biopsy specimens provides a unique role to facilitate the molecular analysis of metastatic tumors, and the identification and validation of therapeutic targets and the discovery and validation of biomarkers that are associated with personalized treatment. This review provides an overview of the development of a metastases and biopsy specimens biobank, analyzes the challenges, and discusses the specific needs regarding this distinct type of biobank development for advancement of personalized medicine.

Keywords: cancer, biospecimen, biorepository, pathology, liquid biopsy, clinical trial

\section{Introduction}

Availability of biomarkers for disease diagnosis and prediction of patient prognosis and therapy promotes the advancement of personalized medicine. Patients are selected based on the presence of particular biomarkers in their samples and receive the most appropriate personalized therapy to achieve satisfactory effects with minimal toxicity. Although much of the recent progress has been made in personalized therapy of primary non-metastatic cancer, personalized medicine for metastatic disease remains a greater challenge, partially due to the complexity of the disease and the lack of research development for targeting metastases. ${ }^{1,2}$ Biobanking high-quality human specimens, such as tissue, blood, and other bodily fluids, and associated patient clinical information provides a fundamental scientific infrastructure for personalized medicine. While current efforts have been made to develop cancer biorepositories around the world for personalized medicine, the majority of biospecimens in these biorepositories are 
primary tumor samples, such as large-scale collection efforts of The Cancer Genome Atlas to catalog the spectrum of mutations present in different tumor types. ${ }^{3,4}$ The procurement, processing, and storage of human metastatic and biopsy specimens are receiving more attention and are important for personalized medicine advancement. Metastasis represents a redundancy of pathways, heterogeneity between primary sites and metastases, and other multifactorial natures, and thus, combination therapies are usually needed for personalized therapy for treating metastatic disease. ${ }^{1}$ Cellular heterogeneity in solid tumors is known, and genetic disparity between primary tumors and metastases has been reported in different types of cancers including colon and prostate cancer. ${ }^{5,6}$ In non-small-cell lung cancer (NSCLC), for example, epidermal growth factor receptor (EGFR) tyrosine kinase inhibitor therapies are targeted to NSCLC harboring EGFR mutations. Studies have shown that EGFR mutation status diverged between primary NSCLCs and their matched distant metastases, ${ }^{7,8}$ which suggests that primary tissue alone is unreliable for therapy decisions in EGFR-targeting therapies. Further, since most patients make follow-up clinical visit to monitor the disease progress and receive further treatment, the profiles of metastatic tumor are important for further targeted therapy. Therefore, besides primary tumor biorepository, biobanking metastasis and biopsy specimen is fundamentally important for personalized medicine. Metastasis often develops in cancer patients despite improvements in surgery, chemotherapy, and radiation therapy, and most metastatic lesions are not the object of surgical resection or even biopsy. Biopsy is usually performed for nonclinical or research purpose, and therefore, it is an important process in clinical trials involving metastatic samples. ${ }^{9-11}$ Further, since it is generally required that all biopsied tissue specimens removed from patient should be examined by a pathologist to receive a pathological diagnosis and evaluation, the scarcity of biopsy samples leftover from diagnosis makes biobanking human metastases and biopsy specimens more challenging and unique.

Proper collection, processing, storage, and tracking of metastases and biopsy specimens are critical components of this distinct type of biobank development and operation. The specific challenges in metastasis and biopsy specimens procurement require significant collaborative efforts between surgeon, pathologist, radiologist, and biobank staff to obtain high-quality biospecimens for banking. Expertise in standardization and quality control, information technology (IT), and laws and regulations, and clinical and pathological knowledge are required for the operations and managements of metastases and biopsy specimen biobank. When the metastases and biopsy specimens biobank is developed to support personalized medicine, actionable results will be returned to clinical practices and therefore would require the highest standards of operations and rigorous quality assurance and quality control process. Certain certification, such as the College of American Pathologists (CAP) accreditation, would be required to maintain the highest operation standard of such patient care-oriented biobank. ${ }^{12,13}$

\section{Procurement and processing of metastases and biopsy specimens}

Metastases and biopsy specimens should be procured from patients with informed consent. Patients should be recruited when they visit clinic or are signed up for certain clinical trials. The informed consent provides a description of the study, an explanation of all procedures, description of benefits and reasonably foreseeable risks, and an explanation that participation is voluntary. ${ }^{14}$ If an informed consent was given when patients are treated for their primary tumor and the consent remains effective, additional consent may not be required for metastatic samples procurement unless patient declined the original informed consent. Certain specific issues such as whether participant samples could be obtained repeatedly for biobanking within the consent duration and whether patients should be recontacted, and whether the metastasis is sampled for purely research or trial purposes, etc are important for procurement of metastases and biopsy specimens and therefore should be included in the consent form. Since biopsy of tumor tissue requires significant clinical efforts and may change clinical management, concerns arise during the biopsy of metastatic tissue collection for future unspecific research. Therefore, biopsy of metastatic tissue should be strictly done using specific clinical research protocols with patient consent. In addition, as new "-omics" technologies, such as next-generation DNA and RNA sequencing, are paving a way toward personalized medicine, and incidental or secondary findings are unavoidable while using these high-throughput technologies. Patient consents should also describe the procedures for handling the incidental findings, and whether the biospecimens and/or test results could be shared with outside physicians and researchers who are not part of the original institution, although the implementation of this practice would be complicated and costly.

Metastatic tumor tissues obtained by routine clinical biopsy or surgical procedures should be examined under supervision of, or examined by, a pathologist. The pathologist plays an essential role in determining tissue allocation for diagnosis and for biobanking. Specifically, when metastatic 
solid tumor tissue is removed from a patient, careful gross examination should be done by a pathologist or pathologist assistant. Dissection of the essential portions is determined by pathologist for routine diagnosis, and additional portions of tissue can be dissected and collected for biobanking without compromising routine pathological diagnosis. For quality control purpose, a portion of biobanked tissue specimen should be embedded in optimal cutting temperature (OCT) compound or fixed in formalin and then embedded in paraffin. A section cut from OCT or formalin-fixed, paraffin-embedded (FFPE) tissue should be stained with hematoxylin and eosin (H\&E) for morphological analysis by a pathologist to evaluate the histological quality of tissue. Biopsy samples collected from solid metastatic tumor should be transported to the pathology lab. At least one needle biopsy core needs to be embedded in OCT or paraffin for quality control purpose and clinical diagnosis. Several additional biopsy samples that are dedicated to biobanking can be snap frozen as soon as they are obtained for biobanking without further pathological examination and diagnosis. Biopsy of primary tumor often occurs before primary cancer treatment and before a diagnosis is firmly established, and thus requires all needle biopsy cores to be examined by a pathologist. Upon completion of diagnosis, redundant needle biopsy cores may be banked for future studies. Therefore, procurement of primary tumor biopsy samples for biobanking is quite different and sometime remains challenging. For procurement of both surgical metastases and biopsy specimens for biobanking, a high speed is essential because a large body of research has demonstrated that prolonged ex vivo ischemia time (ie, the time taken for tissue removal from the patient until snap freezing or fixation in fixative) can compromise biospecimen quality and could potentially impact the research results. ${ }^{15,16}$ In recognizing the effect of ischemia time on biospecimen quality, the American Society of Clinical Oncologist and CAP recently published guidelines to limit the ischemia time to $\leq 60$ minutes for estrogen receptor (ER), progesterone receptor (PR), and Her-2 testing. ${ }^{17,18}$ Although it is challenging at a large medical center with high patient volume, multidisciplinary collaborative efforts should be made to avoid prolonged ischemia time ( $>30$ minutes) on daily biobanking practice. Technically, biopsy specimens can be snap frozen as soon as they are removed from the patient without gross examination, and are relatively easier for processing and handling than surgical metastasis specimens. After biospecimens are stored at $-80^{\circ} \mathrm{C}$ or in liquid nitrogen, repeated freezethaw cycles should be avoided. Large surgically removed metastatic tissue samples that are collected for biobanking should be aliquoted, snap frozen, and stored. Snap freezing of fresh biospecimens should be done because this procedure can inhibit degradation of RNA and protein by slowing the actions of proteases and nucleases, and better maintain the integrity of the biospecimen. The key steps for metastases and biopsy specimens procurement and biobanking are outlined in Table 1. Clinical FFPE tissue blocks may also be collected for biobanking with an institutional review board (IRB)-approved protocol. The corresponding H\&E slides should be reviewed by a pathologist to determine the tumor area and decide the amount of tumor tissue to be collected for biobanking.

Subsequent morphological analysis of frozen or paraffin slides generated from both surgical metastases and biopsy specimens by a pathologist is required. When the collected specimen is of no value for biobanking, such as massive necrosis, or when too little tumor tissue is observed on quality control tissue section, the banked frozen tissue may be discarded.

In addition to biopsy of metastatic solid tumors, liquid biopsy is a relatively new technique with an exponential increase in applications in personalized medicine. Liquid biopsy is isolating liquid biomarker from body fluids, mainly blood. ${ }^{19-21}$ Circulating tumor cells (CTCs) and cell-free circulating tumor DNA (ctDNA) in cancer patients' blood are most interesting targets for liquid biopsy and are becoming a highly sought-after biospecimen type. CTCs are disseminated from primary and/or metastatic tumors throughout the circulatory

Table I Key steps for metastases and biopsy tumor tissue procurement, processing, and storage

Verify patient signed informed consent form.

For metastatic tumor tissues procured from surgery or autopsy, gross check tumor tissue by a pathologist as soon as the procedure allows and record the tissue excision time.

Dissect appropriate portion of tumor and adjacent normal issue for biobanking by a pathologist without compromising routine diagnosis. Make tissue aliquots, embed one or more aliquots in OCT for morphological review by a pathologist for quality assurance and quality control purpose, and snap freeze other aliquots in cryovials in liquid nitrogen by a biobank staff.

For biopsy tumor tissue specimens, record the biopsy time, and embed one needle biopsy core in OCT for morphological review by a pathologist for quality assurance and quality control purpose. Snap freeze other needle biopsy cores in cryovials in liquid nitrogen. Record the time of freezing. Ideally, do not allow $>30$ minutes of ischemia time between the time of excision and time of freezing of tissue sample.

Store all cryovials that contain tissue specimen aliquots at $-80^{\circ} \mathrm{C}$ or in liquid nitrogen vapor for long-term cryo-storage.

Record freezer storage location, biospecimen data, and other relevant patient clinical information into biobank database and update inventory. Abbreviation: OCT, optimal cutting temperature. 
system, while ctDNAs are shed into the bloodstream from dying tumor cells. ${ }^{22}$ CTCs can be detected and isolated via a number of innovative technologies based on the physical or biological properties of CTCs including membrane filter devices, Ficoll centrifugation, CTC microchips, and novel in vivo functionalized and structured Seldinger guidewire technology. ${ }^{19,23}$ Analyses of CTCs can be helpful for understanding the tumor metastatic process and following disease progression. Compared to CTCs analysis, molecular analysis of ctDNA can be used to identify mutations associated with acquired drug assistance in cancer patients and to monitor the disease progression during treatment. ${ }^{21,24}$ In order to avoid the rupture of blood cell membranes and contamination with DNA derived from blood cell nuclei, several special handling procedures for blood collection for ctDNA detection and analysis are required. ${ }^{21}$ Generally, within 2 hours after blood collection, plasma needs to be separated by centrifugation at a low speed to avoid causing cell lysis. ${ }^{25,26}$ Studies have shown that plasma from heparin-stabilized blood or from ethylenediamine tetra-acetate-coated collection tubes is suitable for ctDNA detection and quantification. ${ }^{26,27}$ Although serum can be used for ctDNA analysis, it is reported that serum is less suitable than plasma because of the contamination with DNA from leukocytes during blood coagulation. ${ }^{25,28,29}$ ctDNA can be detected and analyzed based on tumor-specific mutations through real-time polymerase chain reaction (RT-PCR), digital PCR, and next-generation sequencing. ${ }^{29}$ As the development of personalized medicine for cancer patients is dependent on the identification of biomarkers in their sample, and ctDNA and CTCs have significant potential as a biomarker in cancer, liquid biopsy specimens can be a potential alternative of the costly tissue biopsy specimens that are harmfully obtained from metastatic tumors. Several research projects conducted by investigators at our institution are using liquid biopsy to capture ctDNA and CTCs. Blood is taken from patients who enrolled in the projects and processed within 2 hours. ctDNA and CTCs were captured and analyzed or banked for future analysis. As new analytic technologies have evolved, biospecimen types and collection methodologies will continue to be developed as well.

\section{Storage and distribution of metastases and biopsy specimens}

Although currently clinical FFPE tissue blocks and sections are routinely stored at room temperature for official archive, biobanked FFPE tissues for personalized medicine should be stored at $-20^{\circ} \mathrm{C}$ or at a cooler temperature to maintain high-quality nucleic acid and proteins. Fresh frozen and
OCT-embedded metastases and biopsy specimens should be typically stored at $-80^{\circ} \mathrm{C}$ or in liquid nitrogen vapor phase, which can retain a high degree of nucleic acid and protein integrity for biomarker identification and validation. Although isolated CTCs should be stored at $-80^{\circ} \mathrm{C}$ until further processing for DNA and/or RNA extraction, ${ }^{30}$ ctDNA can be stored at $-20^{\circ} \mathrm{C}$ until further analysis. ${ }^{31}$ All biospecimens should be maintained in good condition until distribution for downstream analysis. Several studies have showed that repeated freezing and thawing can lead to significant changes in serum and plasma proteomics and decrease RNA integrity in tissue and blood. ${ }^{32-35}$ Therefore, frozen biospecimens should be aliquoted before they are snap frozen to avoid the repeated freeze-thaw cycles. Although there is very little information available as to whether storage of tissue biospecimens in liquid nitrogen is better than storage at $-80^{\circ} \mathrm{C}$, some studies showed that RNA integrity was reduced in specimens stored at $-70^{\circ} \mathrm{C}$ or $-80^{\circ} \mathrm{C}$ for over 5 years, ${ }^{36,37}$ while no degradation of protein was seen in plasma stored at $-70^{\circ} \mathrm{C}$ for up to 59 months. ${ }^{38}$ Multiple biopsy specimens from the same tumor or multiple aliquots generated from a large surgical metastatic tumor specimen should be stored in two separate locations $\left(-80^{\circ} \mathrm{C}\right.$ and liquid nitrogen vapor) in order to protect the valuable biobank resource from loss. Biospecimens that are housed in the $-80^{\circ} \mathrm{C}$ freezers should be used first. The liquid nitrogen vapor storage serves as a "back-up" that will be used when samples in the $-80^{\circ} \mathrm{C}$ freezer have been exhausted. Secure cryovials such as screw-cap cryovials are recommended for biospecimen containers for long-term, low-temperature storage to reduce contamination from handling. Cryovials that are typically stored at $-80^{\circ} \mathrm{C}$ and in liquid nitrogen vapor phase should be labeled with temperature-resistant cryogenic storage barcoding labels to prevent cracking, peeling, or degrading. Biospecimen type, freezer storage location, and patient information associated with the metastases and biopsy specimens are required to be stored on a secure database system and should be backed up frequently. Barcoding links the biospecimen to the data that are recorded in the database and plays an essential role in tracking samples. Bar codes are suitable for automation or manual operations, provide a tool for reviewing the large quantities of data, and help reduce human errors, which improve efficiency and reduce biospecimen handling cost.

Standard operating procedures (SOPs) should be developed to guide the operations of metastasis and biopsy specimen biobanking activities from procurement through distribution. Some best practices for biorepositories such as International Society for Biological and Environmental Repositories 
(ISBER) best practices for repository and the National Cancer Institute best practices for biospecimen resources can be used as guidelines and references when developing local SOPs. ${ }^{39,40}$ In addition, the CAP biorepository accreditation checklist provides detailed accreditation requirements to ensure that quality management programs are implemented in the biorepository, which can be referenced as well. ${ }^{13}$

High-quality metastases and biopsy specimens along with relevant patient clinical information should be reliable and easily accessible for downstream utilizations. After actionable laboratory results generated from biospecimen are returned to clinicians for delivering personalized therapy to patient, all redundant laboratory results and data can be stored for further translational and clinical research. IT plays an important role in biospecimen data and patient information management. In addition, information management systems are important for tracking biospecimen acquisitions, processing, storage, and distribution as well. Generally, biospecimen type, freezer storage location, patient demographical data (including age, sex, and race), patient clinical data including diagnosis, important laboratory results, for example, ER, PR, and Her-2 status for breast cancer, EGFR mutation for lung cancer, and follow-up outcome data should be captured and stored in the central biobank database. A web-based search engine tool provides the ability to perform customized biospecimen queries, which can significantly reduce the often overwhelming tasks of finding and retrieving biospecimens and make the biobank more easily accessible. ${ }^{41,42}$ However, there are several challenges for IT developers creating systems that are used to manage metastases and biopsy specimens biobank. For example, most metastatic specimens are obtained from patients at follow-up doctor visits, and patient primary diagnosis and follow-up data must be obtained and entered into the database. Different clinicians or medical facilities may be involved in patient treatments over the years. Their medical report on disease progression may be difficult to collect, and standards for diagnostic procedures and criteria may change over time. Thus, extra efforts and steps must be taken to ensure that important data are collected and remain available in the database. The biobank should serve as an honest broker to keep sets of private patient information on a password-protected computer system with support from an institutional secure server. ${ }^{41,43}$ Requests for metastases and biopsy specimens should include a data use agreement as approved by the IRB. The release or disclosure of protected health information to investigators who have IRB-approved protocol for biospecimen usage should consist of only a limited de-identified data set. All released data should have a release code, for example, biospecimen reference number, that allows the biobank personnel to relink the data to the sample and to trace the data's origin.

A multidisciplinary advisory committee would be beneficial to the development and management of metastases and biopsy specimen biobank. The advisory committee should consist of faculty members who represent surgery, pathology, radiology, oncology, translational and clinical science, biostatistics, and bioethicists. The major roles of the advisory committee are to guide polices and SOPs development, review biospecimen requests to define the scientific merit, and prioritize resources to enable fair access to valuable and limited biospecimens. Metastases and biopsy specimen procurement and follow-up clinical data capture would further require multidisciplinary support, adequate staffing, and technical personnel. The infrastructure, investment, and operating cost of this type of biobank would require a significant institutional commitment at the beginning. When the biobanked metastases and biopsy specimens are used for biomarker testing, actionable results may be returned to clinical care, and redundant data and biospecimens may be shared with investigators and pharmaceutical industries. Thus, reimbursement by patient health insurance companies, and chargeback from investigators and pharmaceutical industries may ultimately be required for long-term biobank sustainability. ${ }^{44}$

\section{Quality assurance and quality control of metastases and biopsy specimens}

High-quality metastases and biopsy specimens are of most importance for a successful biobank development. Lowquality biospecimens will likely generate erroneous and misleading data. It has been shown that improper biospecimen handling is one of important factors that contribute to the inaccurate Her-2 diagnostic testing linked to breast cancer therapy. ${ }^{45}$ Therefore, proper biospecimen handling and identity is crucial for high-quality biobank development and management. Detailed documentation of the quality management programs and SOPs ensures that the quality practices on biobanking metastases and biopsy specimen are maintained. Minimal quality control procedures should be performed on biobanked metastases and biopsy specimens, which include morphological analysis of tissue biospecimens and quality measurement of biomolecules that are extracted from those biospecimens. Morphological analysis of biobanked solid metastases and biopsy tissue specimens must be performed by a pathologist to review the histopathological details of H\&E slides that are generated from biobanked tissue 
specimens (Figure 1). Although a single mirror image section can be used for quality control purpose for most surgically removed metastatic tumor specimens, ${ }^{46}$ multiple cryostat sectioning may be required for biopsy specimen and certain types of tumors with infiltrating pattern such as pancreatic and prostate cancer tissues. A top section cut from an OCTembedded frozen tissue block should be stained with $\mathrm{H} \& \mathrm{E}$ and reviewed by a pathologist to determine the amount of cells of interest (eg, tumor cells), and then, a number of sections are cut subsequently and dedicated to nucleic acid or other biomolecular extraction. After that, a bottom section should be cut from the OCT block and stained with H\&E to confirm the presence of cells of interest. Though this is a rigorous histological quality control procedure, it assures that nucleic acid or other biomolecules can be extracted from the cells of interest. The detailed histological quality control reports of the biobanked tissue samples include verification of pathological diagnosis and disease status, evaluation of percentage of tumor, normal, stromal, and necrotic components, and confirmation of the presence of inflammatory cells and necrosis. Specifically, pathological diagnosis of biobanked metastatic tissues should be compared with that of the original primary tumor to confirm the progression of the disease. The histological quality control reports should be stored in the biorepository database associated with the biobanked metastases and biopsy specimens.

Total RNA extracted from metastases and biopsy specimens should be run on a spectrophotometer and an agarose gel. A ratio of spectrophotometric readings at $260 \mathrm{~nm}$ and $280 \mathrm{~nm}$ $\left(A_{260}: A_{280}\right)>1.8$ indicates the acceptable purity of RNA. ${ }^{47}$
A ribosomal RNA (rRNA) $28 \mathrm{~S} / 18 \mathrm{~S}$ ratio of $\geq 2$ indicates high-quality RNA, but generally, a $28 \mathrm{~S}: 18 \mathrm{~S}>1.0$ could be considered to indicate good quality. ${ }^{48}$ Recently, a newly developed automated RNA quality measurement, the RNA integrity number (RIN) or RIN ${ }^{\mathrm{e}}$, has been widely used. ${ }^{49,50}$ The RIN and RIN ${ }^{\mathrm{e}}$ are generated by Agilent Bioanalyzer and Agilent TapeStation software algorithms, respectively. Both RIN and RIN ${ }^{\mathrm{e}}$ are user-independent, automated, and reliable procedures for standardization of RNA quality control. ${ }^{49,51}$ The RIN or $\mathrm{RIN}^{\mathrm{e}}$ values range from 10 to 1 , whereby the intact highest quality RNA is assigned an RIN or RIN ${ }^{\mathrm{e}}$ value of 10 . The profile of high-quality RNA generated by Agilent Bioanalyzer or TapeStation is characterized by two clear, welldefined $28 \mathrm{~S}$ and $18 \mathrm{~S}$ peaks with low noise between the peaks and minimal low-molecular weight noise before the $18 \mathrm{~S}$ peak (Figure 2). Previous reports showed that RNA with an RIN of $\geq 5$ is suitable for RT-PCR analysis, while RNA with an RIN of $\geq 7$ is suitable for microarrays. ${ }^{52-54}$ Since RIN or RIN ${ }^{e}$ value may not accurately indicate the quality of RNA isolated from FFPE tissue, two sets of primers for the upstream end and downstream end of housekeeping genes such as $\beta$-actin, followed by the RT-PCR analysis, are required to assess the quality of FFPE RNA, and a lower ratio of the $3^{\prime}$ end to the $5^{\prime}$ end PCR products indicates higher quality RNA. ${ }^{12}$ The quality of DNA extracted from biobanked metastases and biopsy specimens can be assessed using a Nanodrop to determine the DNA quantity and purity. Similar to RNA measurement, a ratio of spectrophotometric readings at $260 \mathrm{~nm}$ and $280 \mathrm{~nm}\left(A_{260}: A_{280}\right)>1.8$ indicates the acceptable purity of DNA. Several sets of primers of housekeeping genes such as

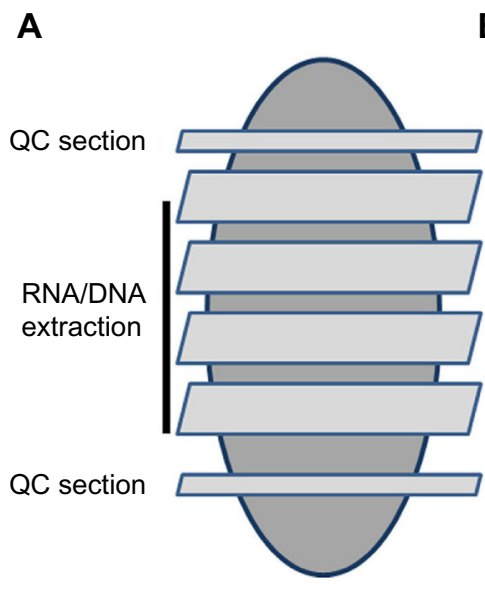

B

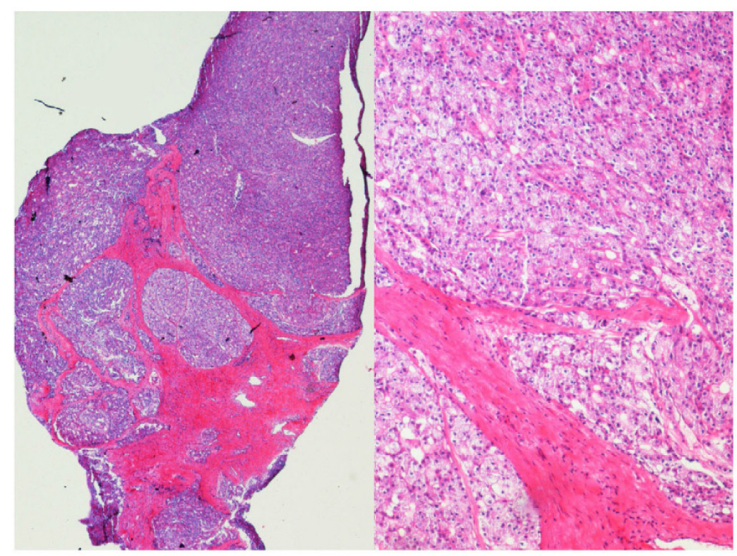

Figure I Tissue histology quality control.

Notes: (A) Serial sections were cut from OCT-embedded biobanked biopsy tissue specimen. The top and bottom slides were stained with H\&E and reviewed by a pathologist. (B) Low-power (left, $\times 4$ ) and high-power field (right, $\times 10)$ of H\&E slide generated from OCT block for histopathological review. Tissue quality was assessed by pathological analysis including confirmation of the histological diagnosis, assessment of tumor purity, and documentation of the presence of normal, stromal, necrotic, and other components.

Abbreviations: OCT, optimal cutting temperature; H\&E, hematoxylin and eosin; QC, quality control. 
A
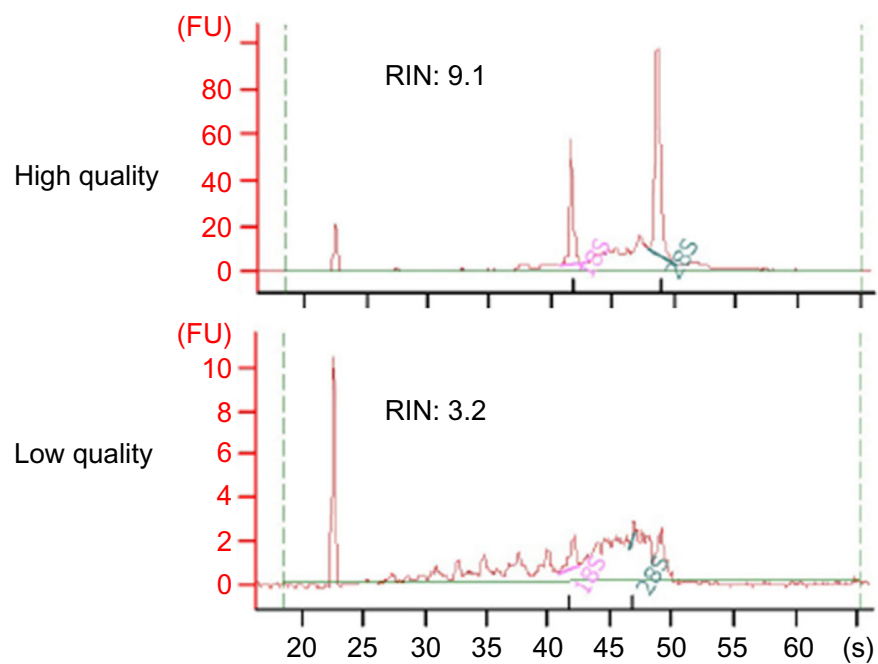

B

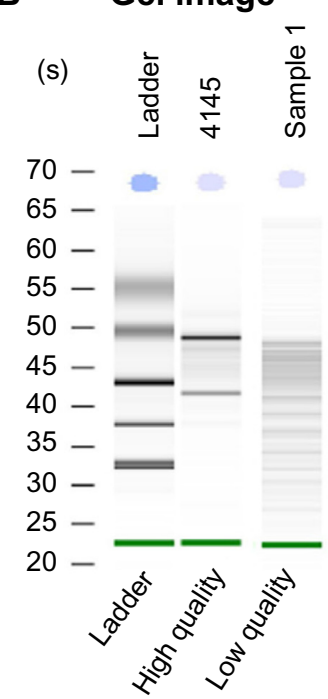

Figure 2 RNA quality control.

Notes: The quality of RNA that was extracted from metastases and biopsy specimens was analyzed using the Agilent Bioanalyzer. (A) High-quality RNA (upper panel) shows two clear and well-defined peaks of I8S and 28S rRNA with a high RIN (RIN =9.1), and minimal low-molecular weight noise between the I8S and 28S peaks and before the I8S peak, while degraded RNA (lower panel) shows a smaller $28 \mathrm{~S}$ than $18 \mathrm{~S}$ peak with a low RIN (RIN =5.7), and the presence of amounts of low-molecular weight noise before I8S and between I8S and 28S peaks. (B) Pseudo gel image of the same RNA in (A and B) generated by Agilent Bioanalyzer.

Abbreviations: rRNA, ribosomal RNA; RIN, RNA integrity number.

$\beta$-globin followed by the PCR can be used to amplify different length fragments to assess the quality of DNA, and the larger maximum amplicon size indicates higher DNA quality. More recently, Agilent TapeStation was developed and assess both quantity and quality of DNA in a single step..$^{55}$ On the DNA profile generated by Agilent TapeStation, high-quality DNA runs as a clearly defined single band, while degraded DNA runs as a smear lacking a sharp band (Figure 3). Although several different protein assays such as bicinchoninic acid assay, Lowry protein assay, sodium dodecyl sulfate polyacrylamide gel electrophoresis followed by Coomassie Blue staining, immunohistochemical staining, and Western blotting can
A

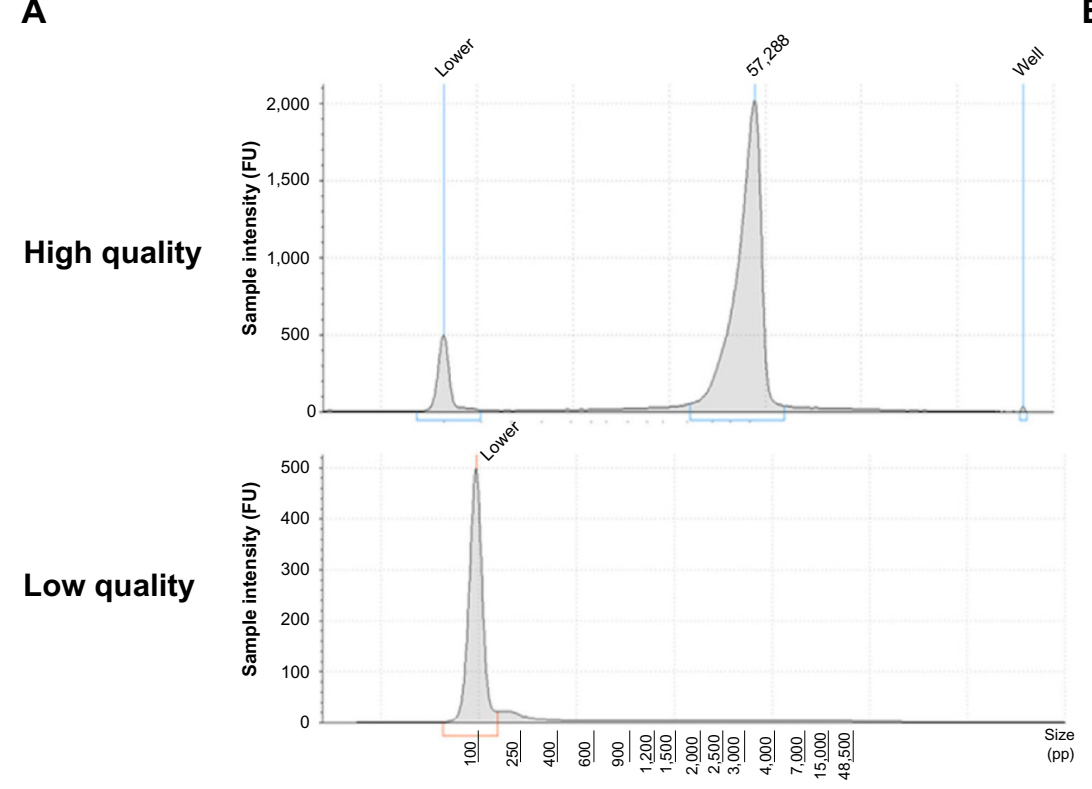

B

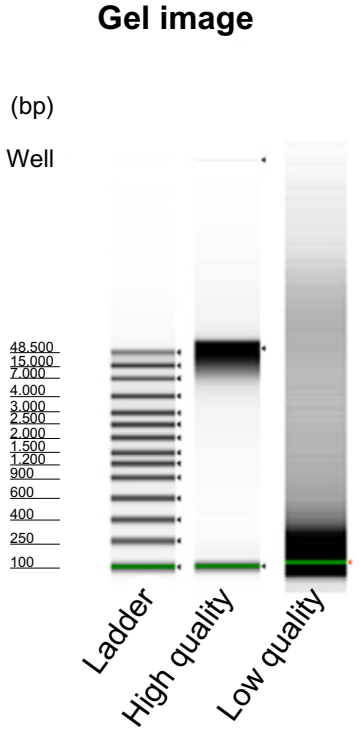

Figure 3 DNA quality control.

Notes: The quality of DNA that was extracted from metastases and biopsy specimens was analyzed using the Agilent TapeStation. (A) High-quality DNA (upper panel) shows a single large peak with minimal low-molecular weight noise, while degraded DNA (lower panel) shows a very low peak with amounts of low-molecular weight noise. (B) Gel image of the same DNA in (A and B) generated by Agilent TapeStation. 
be used to assess the protein concentration and integrity, the protein quality evaluations are generally not cost effective or efficient. Because the metastases and biopsy specimens are valuable, it would be best to leave to the end user investigators the assessment of the protein quality based on their specific downstream applications and analytical processes. In addition to the quality control of the metastases and biopsy specimens, documents and records control, internal audits, and corrective and preventive actions are important as well in the operations and managements of such valuable and distinct biobank.

\section{Cancer pathology in biobanking metastases and biopsy specimens}

Pathologist plays an important role in operations and managements of tissue biobank. ${ }^{56-58}$ Because solid cancer tissue specimens are composed of heterogeneous cell types including tumor cells, adjacent normal cells, stromal cells, inflammatory cells, and other components, histological quality control of metastases and biopsy tissue specimens must be performed by a pathologist. In addition to the unique role in solid tissue histological quality control and evaluation, because of pathologists' role in examining human tissues for diagnosis or therapy of diseases, only pathologists or pathologist assistants are qualified to gross check the solid tissue specimen and dissect the appropriate portion of tissue for pathological diagnosis and select other portion of tissue for biobanking without compromising routine patient care. Biobanking metastases and biopsy tissue specimens in support of personalized medicine requires high-quality DNA and/or RNA to be extracted from biobanked specimens for downstream biomarkers discovery and development. Results generated through the varied sensitivity of molecular biology technologies may represent dominant cell types but not specific type of cells of interest (eg, tumor cells) because of the heterogeneous nature of solid tumor tissues; metastases and biopsy tissue specimens often require subsequent procedures including macrodissection or laser capture microdissection to reduce the microheterogeneity within the tissue specimens and enrich the purity of specific cells of interest (eg, tumor cells) prior to RNA/DNA/protein extraction for downstream applications. ${ }^{59}$ Pathologist's expertise is required for the macro- and microdissection process to recognize the needed specific types of cells for harvest.

Certain tissue-based technologies such as tissue microarray (TMA), immunohistochemistry, and in situ hybridization are becoming common services and practices within a tissue biobank. TMA allows rapid and simultaneous morphological assessment in large sets of tissue specimens on a single TMA slide. Combined with immunohistochemical staining, in situ hybridization, and fluorescent in situ hybridization, TMA provides a powerful tool for high-throughput screening and validation for biomarker studies. ${ }^{60,61}$ TMA blocks are prepared by transferring tumor tissue from many paraffin or OCT blocks to a recipient block. Metastatic tissue samples can be constructed with primary tumor on a single TMA block, which provide a powerful way to monitor biomarker changes during tumor progression and metastasis. For TMA construction, an H\&E slide generated from original paraffin or OCT blocks should be reviewed by a pathologist to identify the proper tumor area for transferring to recipient TMA block. After TMA construction is completed, slides generated from TMA block that contains many samples from multiple different donor blocks should be reviewed by a pathologist for subsequent analyses as well. Because of the requirement of cancer pathology in tissue biobank, pathologist plays a critical role in operations and managements of metastases and biopsy specimens biobank.

\section{Whole-genome and whole-exome sequencing and managing incidental findings}

The ability to sequence each tumor's whole genome and whole exome quickly and inexpensively by next-generation sequencing is rapidly becoming the standard of molecular genetic assays and opens the way to new avenues in personalized medicine. ${ }^{10,62}$ Actionable mutations in multiple genes and pathways detected in metastases and biopsy specimens by next-generation sequencing at an affordable cost tremendously facilitate the selection of targeted therapies. After actionable results are returned to oncologists for patient personalized therapy, all redundant data generated by nextgeneration sequencing using biobanked metastases and biopsy specimens should be stored in a secure central database for future research use. These existing sequencing data along with patient clinical information provide an invaluable data resource for new discovery without repeated biospecimens acquisition and sequencing, which tremendously promises the efficiency and effectiveness of translational and clinical research. The storage of massive genomic data is an important issue, especially when primary and metastasis parings are considered. Separate database dedicated to the processed sequencing data storage may be needed and should be integrated with the biorepository database and other Laboratory Information Management System. In order to protect patient privacy and confidentiality, future analysis of these data beyond the scope of the original study should be incorporated into informed consent documents and would need a separate IRB approval. Combining the whole-genome and whole-exome sequencing 
and metastases and biopsy specimens biobank increasingly plays a critical role in identifying genetic variation and the associations between the genetic variation and drug efficacy and clinical outcomes.

Because the high-throughput next-generation sequencing technology has the capacity to generate massive amounts of data, incidental or secondary findings that are not related to the original data of interest but may be potentially clinically relevant are unavoidable and increasing. Whether or not the incidental or secondary findings should be returned to oncologists and/or patients for further treatment consideration is still controversial. However, it is recommended that certain findings and results that reveal an established and substantial risk of serious health, test-validated by a Clinical Laboratory Improvement Amendments-certified laboratory, and are clinically actionable be returned to consented patients. ${ }^{63,64}$ Subsequently, the American College of Medical Genetics and Genomics recommended a minimum list of conditions, gene, and variants for return of incidental findings in clinical sequencing. ${ }^{65}$ More recently, the Presidential Commission for the Study of Bioethical Issues, a White House advisory panel, recommended that researchers, clinicians, and direct-to-consumer firms that generate genomic, imaging, or other types of biomedical data should plan to encounter incidental findings and should develop a plan to communicate with patients, study participants, and consumers about how these findings will be handled. ${ }^{66}$ Because of these recommendations, although the process of returning incidental findings to participants is complicated and incurs significant costs, a responsible mechanism for returning the incidental findings or developing a policy to determine how to handle these findings should be in place for biobanking metastases and biopsy specimens in support of personalized medicine.

\section{Conclusion}

Biomarkers discovery and development in oncology are advancing rapidly and have facilitated the development of new drug targets and targeted treatment strategies. Biomarkers that are identified in patient samples have enhanced the ability of oncologists to deliver targeted therapy with minimal toxicity, which is beginning to make personalized medicine a reality in oncology. Biomarker-driven treatment plan based on the molecular profile of the tumor has been shifted as standard of care for patient with advanced-stage cancers with metastases, partially owing to the high-throughput next-generation sequencing, which enables the rapid and comprehensive whole-genome and whole-exome analysis of individual patient tumor even from tiny tumor biopsy specimen. Biobanking metastatic and biopsy samples and their derivatives therefore plays an important role in new biomarker discovery and provides a foundation for the development of novel personalized medicines in oncology that benefit patients with advanced metastatic disease. Procuring and storing patient metastases and biopsy specimens and their derivatives for clinically relevant molecular testing is a clearly exciting movement but remains challenging. Procurement of metastases and biopsy specimens requires necessary multidisciplinary efforts including surgery, pathology, radiology, and biobank. Storing and distributing of those biospecimens require the highest standard ethical practices and rigorous quality assurance and quality control procedures. Pathologist and efficient IT database are crucial in such distinct biobank development and management. It is also critical to have a large collection of metastases and biopsy specimens with relevant patient clinical data for effective biomarkers development in a personalized medicine setting. Data generated from biobanked metastases and biopsy specimens by innovative high-throughput next-generation sequencing should be stored and reused for future studies. However, a responsible mechanism to handle the incidental or secondary findings should be in place. As personalized medicine expands, biobanking metastases and biopsy samples will be a necessary and standard practice in the treatment of cancer patients, especially for patients suffering from advanced diseases.

\section{Disclosure}

The authors declare that there are no conflict of interests relevant to the publication of this paper.

\section{References}

1. Marino N, Woditschka S, Reed LT, et al. Breast cancer metastasis: issues for the personalization of its prevention and treatment. Am J Pathol. 2013;183(4):1084-1095.

2. Basik M, Aguilar-Mahecha A, Rousseau C, et al. Biopsies: nextgeneration biospecimens for tailoring therapy. Nat Rev Clin Oncol. 2013;10(8):437-450.

3. Cancer Genome Atlas Research Network. Comprehensive molecular characterization of clear cell renal cell carcinoma. Nature. 2013; 499(7456):43-49.

4. Cancer Genome Atlas Research Network. Integrated genomic analyses of ovarian carcinoma. Nature. 2011;474(7353):609-615.

5. Albanese I, Scibetta AG, Migliavacca M, et al. Heterogeneity within and between primary colorectal carcinomas and matched metastases as revealed by analysis of Ki-ras and 553 mutations. Biochem Biophys Res Commun. 2004;325(3):784-791.

6. Stapleton AM, Timme TL, Gousse AE, et al. Primary human prostate cancer cells harboring p53 mutations are clonally expanded in metastases. Clin Cancer Res. 1997;3(8):1389-1397.

7. Gow CH, Chang YL, Hsu YC, et al. Comparison of epidermal growth factor receptor mutations between primary and corresponding metastatic tumors in tyrosine kinase inhibitor-naive non-small-cell lung cancer. Ann Oncol. 2009;20(4):696-702. 
8. Kalikaki A, Koutsopoulos A, Trypaki M, et al. Comparison of EGFR and K-RAS gene status between primary tumours and corresponding metastases in NSCLC. Br J Cancer. 2008;99(6):923-929.

9. Jamal-Hanjani M, Hackshaw A, Ngai Y, et al. Tracking genomic cancer evolution for precision medicine: the lung TRACERx study. PLoS Biol. 2014;12(7):e1001906.

10. Roychowdhury S, Iyer MK, Robinson DR, et al. Personalized oncology through integrative high-throughput sequencing: a pilot study. Sci Transl Med. 2011;3(111):111ra121.

11. Hong MK, Sapre N, Phal PM, et al. Percutaneous image-guided biopsy of prostate cancer metastases yields samples suitable for genomics and personalised oncology. Clin Exp Metastasis. 2014;31(2):159-167.

12. Liu A. Developing an institutional cancer biorepository for personalized medicine. Clin Biochem. 2014;47(4-5):293-299.

13. College of American Pathologists. Biorepository Checklist CAP Accreditation Program; 2013. Available from: http:/www.cap.org/apps/docs/ laboratory_accreditation/checklists/new/biorepository_checklist.pdf. Accessed October 10, 2014

14. Beskow LM, Friedman JY, Hardy NC, Lin L, Weinfurt KP. Developing a simplified consent form for biobanking. PLoS One. 2010; 5(10):e13302.

15. Dash A, Maine IP, Varambally S, Shen R, Chinnaiyan AM, Rubin MA. Changes in differential gene expression because of warm ischemia time of radical prostatectomy specimens. Am J Pathol. 2002;161(5): 1743-1748.

16. Holzer TR, Fulford AD, Arkins AM, et al. Ischemic time impacts biological integrity of phospho-proteins in PI3K/Akt, Erk/MAPK, and p38 MAPK signaling networks. Anticancer Res. 2011;31(6):2073-2081.

17. Wolff AC, Hammond ME, Hicks DG, et al; for American Society of Clinical Oncology; College of American Pathologists. Recommendations for human epidermal growth factor receptor 2 testing in breast cancer: American Society of Clinical Oncology/College of American Pathologists clinical practice guideline update. J Clin Oncol. 2013;31(31):3997-4013.

18. Hammond ME, Hayes DF, Wolff AC, Mangu PB, Temin S. American society of clinical oncology/college of American pathologists guideline recommendations for immunohistochemical testing of estrogen and progesterone receptors in breast cancer. J Oncol Pract. 2010;6(4): 195-197.

19. Alix-Panabières C, Pantel K. Circulating tumor cells: liquid biopsy of cancer. Clin Chem. 2013;59(1):110-118.

20. Alix-Panabières $C$, Pantel K. Real-time liquid biopsy: circulating tumor cells versus circulating tumor DNA. Ann Transl Med. 2013;1(2):18.

21. Rolfo C, Castiglia M, Hong D, et al. Liquid biopsies in lung cancer: the new ambrosia of researchers. Biochim Biophys Acta. 2014;1846(2): 539-546.

22. Haber DA, Velculescu VE. Blood-based analyses of cancer: circulating tumor cells and circulating tumor DNA. Cancer Discov. 2014;4(6): 650-661.

23. Saucedo-Zeni N, Mewes S, Niestroj R, et al. A novel method for the in vivo isolation of circulating tumor cells from peripheral blood of cancer patients using a functionalized and structured medical wire. Int J Oncol. 2012;41(4):1241-1250.

24. Murtaza M, Dawson SJ, Tsui DW, et al. Non-invasive analysis of acquired resistance to cancer therapy by sequencing of plasma DNA. Nature. 2013;497(7447):108-112.

25. Umetani N, Hiramatsu S, Hoon DS. Higher amount of free circulating DNA in serum than in plasma is not mainly caused by contaminated extraneous DNA during separation. Ann N Y Acad Sci. 2006;1075: 299-307.

26. Su YH, Wang M, Brenner DE, Norton PA, Block TM. Detection of mutated K-ras DNA in urine, plasma, and serum of patients with colorectal carcinoma or adenomatous polyps. Ann N Y Acad Sci. 2008;1137: 197-206.

27. Diehl F, Schmidt K, Durkee KH, et al. Analysis of mutations in DNA isolated from plasma and stool of colorectal cancer patients. Gastroenterology. 2008;135(2):489-498.
28. Gahan PB, Swaminathan R. Circulating nucleic acids in plasma and serum. Recent developments. Ann N Y Acad Sci. 2008;1137:1-6.

29. Benesova L, Belsanova B, Suchanek S, et al. Mutation-based detection and monitoring of cell-free tumor DNA in peripheral blood of cancer patients. Anal Biochem. 2013;433(2):227-234.

30. Barbazán J, Alonso-Alconada L, Muinelo-Romay L, et al. Molecular characterization of circulating tumor cells in human metastatic colorectal cancer. PLoS One. 2012;7(7):e40476.

31. Lebofsky R, Decraene C, Bernard V, et al. Circulating tumor DNA as a non-invasive substitute to metastasis biopsy for tumor genotyping and personalized medicine in a prospective trial across all tumor types. $\mathrm{Mol}$ Oncol. 2015;9(4):783-790.

32. Sherwood KR, Head MW, Walker R, Smith C, Ironside JW, Fazakerley JK. RNA integrity in post mortem human variant Creutzfeldt-Jakob disease (vCJD) and control brain tissue. Neuropathol Appl Neurobiol. 2011;37(6):633-642.

33. Atz M, Walsh D, Cartagena $P$, et al; Members of National Institute of Mental Health Conte Center and Pritzker Neuropsychiatric Disorders Research Consortium. Methodological considerations for gene expression profiling of human brain. J Neurosci Methods. 2007;163(2):295-309.

34. Kopreski MS, Benko FA, Kwak LW, Gocke CD. Detection of tumor messenger RNA in the serum of patients with malignant melanoma. Clin Cancer Res. 1999;5(8):1961-1965.

35. Baumann S, Ceglarek U, Fiedler GM, Lembcke J, Leichtle A, Thiery J. Standardized approach to proteome profiling of human serum based on magnetic bead separation and matrix-assisted laser desorption/ionization time-of-flight mass spectrometry. Clin Chem. 2005;51(6):973-980.

36. Leonard S, Logel J, Luthman D, Casanova M, Kirch D, Freedman R. Biological stability of mRNA isolated from human postmortem brain collections. Biol Psychiatry. 1993;33(6):456-466.

37. Chu TY, Hwang KS, Yu MH, Lee HS, Lai HC, Liu JY. A research-based tumor tissue bank of gynecologic oncology: characteristics of nucleic acids extracted from normal and tumor tissues from different sites. Int J Gynecol Cancer. 2002;12(2):171-176.

38. Lewis MR, Callas PW, Jenny NS, Tracy RP. Longitudinal stability of coagulation, fibrinolysis, and inflammation factors in stored plasma samples. Thromb Haemost. 2001;86(6):1495-1500.

39. National Cancer Institute. 2100 NCI Best Practices for Biospecimen Resource. Available from: http://biospecimens.cancer.gov/ bestpractices/2011-NCIBestPractices.pdf. Accessed March 10, 2015.

40. International Society for Biological and Environmental Repositories. 2012 best practices for repositories collection, storage, retrieval, and distribution of biological materials for research. Biopreserv Biobank. 2012;10(2):79-161.

41. Amin W, Kang HP, Egloff AM, et al. An informatics supported webbased data annotation and query tool to expedite translational research for head and neck malignancies. BMC Cancer. 2009;9:396.

42. Anderson N, Ponko S, Black T, et al. Biotrust: a comprehensive system for acquiring and distributing biospecimens. AMIA Jt Summits Transl Sci Proc. 2013;2013:4.

43. Amin W, Parwani AV, Schmandt L, et al. National Mesothelioma Virtual Bank: a standard based biospecimen and clinical data resource to enhance translational research. BMC Cancer. 2008;8:236.

44. McDonald SA, Watson MA, Rossi J, Becker CM, Jaques DP, Pfeifer JD. A new paradigm for biospecimen banking in the personalized medicine era. Am J Clin Pathol. 2011;136(5):679-684.

45. Wolff AC, Hammond ME, Schwartz JN, et al; for American Society of Clinical Oncology; College of American Pathologists. American Society of Clinical Oncology/College of American Pathologists guideline recommendations for human epidermal growth factor receptor 2 testing in breast cancer. J Clin Oncol. 2007;25(1):118-145.

46. Grizzle WE, Sexton KC, Bell WC. Quality assurance in tissue resources supporting biomedical research. Cell Preserv Technol. 2008;6(2): 113-118.

47. Manchester KL. Use of UV methods for measurement of protein and nucleic acid concentrations. Biotechniques. 1996;20(6):968-970. 
48. Imbeaud S, Graudens E, Boulanger V, et al. Towards standardization of RNA quality assessment using user-independent classifiers of microcapillary electrophoresis traces. Nucleic Acids Res. 2005; 33(6):e56.

49. Schroeder A, Mueller O, Stocker S, et al. The RIN: an RNA integrity number for assigning integrity values to RNA measurements. BMC Mol Biol. 2006; 7:3.

50. Hostetter G, Collins E, Varlan P, et al. Veterinary and human biobanking practices: enhancing molecular sample integrity. Vet Pathol. 2014; 51(1):270-280.

51. Padmanaban A. RNA Quality Control using the Agilent 2200 TapeStation System: Assessment of the RINe Quality Metric; 2012. Agilent technologies application notes, Santa Clara.

52. Fleige S, Pfaffl MW. RNA integrity and the effect on the real-time qRT-PCR performance. Mol Aspects Med. 2006;27(2-3):126-139.

53. Raman T, O'Connor TP, Hackett NR, et al. Quality control in microarray assessment of gene expression in human airway epithelium. BMC Genomics. 2009;10:493.

54. Opitz L, Salinas-Riester G, Grade M, et al. Impact of RNA degradation on gene expression profiling. BMC Med Genomics. 2010;3:36.

55. Guettouche T. Genomic DNA Analysis with the Agilent 2200 TapeStation System and Agilent Genomic DNA ScreenTape; 2013. Agilent Technologies Application Notes, Santa Clara.

56. Bevilacqua G, Bosman F, Dassesse T, et al. The role of the pathologist in tissue banking: European Consensus Expert Group Report. Virchows Arch. 2010;456(4):449-454.

57. Hainaut P, Caboux E, Bevilacqua G, et al. Pathology as the cornerstone of human tissue banking: European consensus expert group report. Biopreserv Biobank. 2009;7(3):157-160.
58. Grizzle WE, Woodruff KH, Trainer TD. The pathologist's role in the use of human tissues in research - legal, ethical, and other issues. Arch Pathol Lab Med. 1996;120(10):909-912.

59. Liu A. Laser capture microdissection in the tissue biorepository. J Biomol Tech. 2010;21(3):120-125.

60. Kononen J, Bubendorf L, Kallioniemi A, et al. Tissue microarrays for high-throughput molecular profiling of hundreds of specimens. Nat Med. 1998;4(7):844-847.

61. Tzankov A, Went P, Zimpfer A, Dirnhofer S. Tissue microarray technology: principles, pitfalls and perspectives - lessons learned from hematological malignancies. Exp Gerontol. 2005;40(8-9):737-744.

62. Dewey FE, Grove ME, Pan C, et al. Clinical interpretation and implications of whole-genome sequencing. JAMA. 2014;311(10):1035-1045.

63. Wolf SM, Crock BN, Van Ness B, et al. Managing incidental findings and research results in genomic research involving biobanks and archived data sets. Genet Med. 2012;14(4):361-384.

64. Wolf SM, Lawrenz FP, Nelson CA, et al. Managing incidental findings in human subjects research: analysis and recommendations. J Law Med Ethics. 2008;36(2):219-248.

65. Green RC, Berg JS, Grody WW, et al; for American College of Medical Genetics and Genomics. ACMG recommendations for reporting of incidental findings in clinical exome and genome sequencing. Genet Med. 2013;15(7):565-574.

66. The Presidential Commission for the Study of Bioethical Issues. Anticipate and Communicate: Ethical Management of Incidental and Secondary Findings in the Clinical, Research, and Direct-to-Consumer Contexts. Washington DC; 2013. Available from: http://www.bioethics. gov. Accessed March 12, 2015.
Journal of Biorepository Science for Applied Medicine

\section{Publish your work in this journal}

The Journal of Biorepository Science for Applied Medicine is an international, peerreviewed, open access journal that focuses on new developments and advances in the emerging and evolving field of biorepository science. This includes biospecimen procurement, processing, preservation, and banking for application to applied medicine. The Journal invites submission of manuscripts which address these aspects in addition to systems logic, clinical throughput and ethical issues pertaining to application of

\section{Dovepress}

biorepositories and their effects on clinical medicine. The journal is characterized by the rapid reporting of reviews, original research, methodologies, technologies and analytics in this subject area. The manuscript management system is completely online and includes a very quick and fair peer-review system, which is all easy to use. Visit http:/ www.dovepress.com/testimonials.php to read real quotes from published authors. 